\title{
Game Theoretic Analysis of Network Dimensioning Strategies in Differentiated Services Networks
}

\author{
Péter Füzesi, Attila Vidács \\ High Speed Networks Laboratory, Dept. of Telecommunications and Telematics, \\ Budapest University of Technology and Economics, \\ Magyar Tudósok körútja 2, H-1117, Budapest, Hungary. \\ Tel: +361 463 2125, Fax: +361 4633107 \\ E-mail: \{fuzesi, vidacs\}@ttt-atm.ttt.bme.hu
}

\begin{abstract}
In the future Internet, introducing service differentiation the quality guarantees seem to have more importance. Beyond the basic performance parameters such as loss probability, delay, jitter, etc., the QoS network dimensioning problem requires the knowledge of the total utility experienced by the users. Considering the users as players competing for the network resources, the Game Theory seems to be a useful tool for describing the behavior of the system. Applying the rules of the noncooperative games where the players are selfish and act individually, Nash equilibria can be used to determine the "rest points" of the system. Analyzing these points by calculating the overall utility gained by the users and the overall income of the network operator, different network dimensioning strategies can be evaluated. This paper introduces evaluation of typical dimensioning strategies in different domain control setups with diverse user expectations in terms of $\mathrm{QoS}$ and price.

Keywords- Differentiated Services, QoS, Nash equlibria, noncooperative games, network dimensioning, pricing
\end{abstract}

\section{INTRODUCTION}

Today's Internet provides "best effort" data transferring mechanisms among any nodes of the network. Numerous services have emerged to use this possibility, e-mail services, FTP, WWW browsing have exploited the advantages of IP (Internet Protocol) based packet forwarding solutions, such as TCP (Transmission Control Protocol). However, without service differentiation, all of the packet flows get the same Quality of Service (QoS). This is an implication of the philosophy of early packet-switched networks, where each packet is treated equally. The routers can guarantee only the "best effort" forwarding of the packets to their destinations.

Introducing QoS management in the Internet, service differentiation becomes an important issue. Beyond the common services using elastic, adaptive traffic (such as web browsing, FTP, etc.), the real-time services (such as multimedia applications) raised new requirements against the network [12]. The delay, jitter, loss probability, etc., have become significant parameters describing a service. The QoS is described by a set of these parameters. Giving bounds (i.e., QoS guarantees) for each parameter, different service classes can be defined.

Today, pricing of the Internet is mostly based on flat rate tariffs for the users. The QoS guarantees can change this situation since the "guarantees" will represent the new value, this is what the users pay for to the network operators.

It is worth examining the kind of changes that can occur introducing quality guarantees from the user's perspective. First, it is obvious that better quality improves the utility experienced by the users. Below a certain level the quality can be unacceptable. For example, users can decide what the worst quality is which they can still tolerate while watching a movie. Secondly, it can be assumed that better quality is more expensive since it consumes more network resources. It can also influence the experienced utility, which implies that the users always want to get satisfactory QoS in the cheapest way. In general, while dimensioning a network, the performance parameters such as loss probability, delay, etc., mean only a subset of the important quantities to be investigated. The total utility, which is calculated by looking at the individual "feelings" of the users, reflects their general attitude to the network.

\section{A. Architectures offering $Q o S$}

The problem of offering QoS on IP networks launched exhaustive research. In the best effort Internet, the same-serviceto-all model makes it impossible to introduce service differentiation, which is strongly required for offering QoS for different demands. Two basic approaches have emerged to solve this problem. The Integrated Services [13] architecture provides service differentiation for each individual flow, while the Differentiated Services [2] model defines service classes for flow aggregates.

\section{A.1 The IntServ model}

The Integrated Services (IntServ) architecture is based on per flow treatment since it ensures QoS for each individual traffic flow. Each flow can request a specific level of service by defining given QoS parameters, e.g., maximum tolerable endto-end delay, minimum service rate, etc. Admission control is performed at each node on the route of the flow, usually using a resource reservation protocol, such as RSVP [3]. Once a reservation succeeds, the required QoS is guaranteed. The main advantage of the model is the strict service differentiation on flow level which results in hard QoS guarantees for the accepted requests. However, because of the expected large number of flows in networks such as the Internet, the IntServ model does not seem to scale well.

\section{A.2 The DiffServ model}

The Differentiated Services (DiffServ) architecture seems to be promising and more realistic in the near future [2]. In this 
case, the per flow treatment is only applied at the border of the network domain. Within the domain, different service classes can be defined by certain QoS parameters. After mapping the QoS requirements to a previously defined service class, the packets belonging to the flow are marked, composing aggregates of flows in this way. The treatment of the packets is based on these aggregates ensuring less processing overhead and more scalability.

The admission control is performed on the border of the domains. It can be based on signaling, similarly to the IntServ model or based on the decisions of a Bandwidth Broker [9] which is aware of the actual available free resources within a domain.

\section{B. Game theoretic approach of user behavior}

In a game theoretic approach the users can be considered as players competing for network resources. The users of the network play non-cooperative game against each other which means that the users make decisions individually following their own intentions trying to maximize their experienced utility [7]. Those situations, where none of the players can improve the experienced utility by individual actions are called Nash equilibria [4], [1]. The Nash equilibrium is one of the most important concepts which can be used for examining the non-cooperative games since it is set without any external interference.

In general, within a certain network configuration more situations can exist which are Nash equilibria. Moreover, the existence of Nash equilibrium does not necessarily imply optimal resource consumption or maximum overall utility experienced by the users.

\section{Motivation}

In this article we investigate different network dimensioning strategies where the goal is to optimize the network resource consumption and to fulfill different economic demands in Differentiated Services networks. Evaluating the user behavior in the resulted configurations using game theoretic approach is an issue as well.

First, in section II the basic model and the background of the game theoretic approach is introduced. After giving an extension to the model and summarizing the investigated domain control scenarios in section III, different methods are introduced for sizing the capacities of the service classes in IV. In section $\mathrm{V}$, evaluation is given on the dimensioning strategies in different control scenarios.

\section{BACKGROUND}

\section{A. Game theoretic aspect}

Several articles have already dealt with the game theoretic approach of characterizing the behavior of the network in a non-cooperative environment [10], [11]. The routing methods
[8] and the resource allocation [7] have been investigated from this aspect as well.

\section{B. The basic model}

Park et al. have introduced a model framework which focuses on the user behavior when different service classes are available [10], [11]. In the model each service class has a QoS function which determines the QoS parameters depending on the class load. Each user has a bandwidth requirement and a certain threshold which determines the QoS parameters (e.g., packet loss, delay) which can be tolerated by the user. In the original model, the strategy of a user is defined by the way the available service classes are used. That is the way the total bandwidth requirement is distributed among them. The resource allocation matrix $\Lambda$ shows the strategies of the users as follows:

$$
\Lambda=\left[\lambda_{i j}\right], \quad i \in[1, m], \quad j \in[1, n],
$$

where we consider $m$ users and $n$ service classes, $\lambda_{i j}$ denotes the traffic amount sent to service class $j$ by user $i$ (which means a bandwidth requirement as well). The total bandwidth requirement $\lambda_{i}$ of user $i$ is given by:

$$
\lambda_{i}=\sum_{j=1}^{n} \lambda_{i j} .
$$

As a simplification of the model, also mentioned by Park et $a l$, in the case of "unsplittable games" the users cannot smooth the traffic among the given service classes. The strategy is defined by the choice, so that to which particular class the whole traffic is sent. In this case, the goal is to find a satisfactory service class where the rendered QoS is below the users' threshold after allocating their bandwidth requirement.

Without loosing the generality of the model we believe that the latter method shows the typical way of resource allocation. In this article, we consider the unsplittable case only, where a user has to choose only one service class, so $\lambda_{i}=\lambda_{i j}$ where $j$ is the chosen class. The total amount of traffic sent to service class $j, q_{j}$, is given by:

$$
q_{j}=\sum_{i=1}^{m} \lambda_{i j}
$$

Function $c_{j}\left(q_{j}\right)$ is defined as the QoS provided by class $j$ at load level $q_{j}$. In this model, a scalar value is used for describing the QoS (e.g., loss rate). It is assumed, that $c_{j}$ is monotonically increasing and bigger $c_{j}\left(q_{j}\right)$ means worse QoS.

Each user has a QoS threshold parameter $\Theta_{i}$ corresponding to the maximum QoS value which can still be tolerated by the user. Based on the model of Park et al. a utility function $U_{i j}$ is defined for each user choosing service class $j$ which determines the utility experienced by user $i$ as zero, if the experienced QoS is above the threshold $\Theta_{i}$, and $\lambda_{i j}$ otherwise:

$$
U_{i j}=\left\{\begin{array}{ll}
\lambda_{i j}, & \text { if } \Theta_{i} \geq c_{j}\left(q_{j}\right) \\
0, & \text { if } \Theta_{i}<c_{j}\left(q_{j}\right)
\end{array} .\right.
$$


As the players try to improve their individual lots, they try to find a service class which satisfies their QoS requirements by maximizing the experienced utility:

$$
U_{i}^{\max }=\max _{j}\left(U_{i j}\right) .
$$

\section{Algorithm to reach Nash equilibria}

Park et al. suggest a simple algorithm to reach Nash equilibria assuming only one QoS parameter [10]. After ordering the users based on their QoS threshold as defined in (6), the rule is that the most "insensitive" user steps first, who can tolerate the greatest loss of quality.

$$
\forall i<j, \quad \Theta_{i} \geq \Theta_{j} .
$$

Considering the second rule, which states that a user does not step if no step can improve its individual utility, the algorithm ensures that the sensitive users will not affect the utility of the insensitive users. It is proven that this algorithm leads to Nash equilibria quickly.

\section{THE PROPOSED MODEL}

Our extension to the basic model and the examined networking scenarios are given next.

\section{A. Pricing parameter}

Let us assume an order among the service classes so that the first service class can offer the worst QoS and the last service class provides the best QoS. If $c_{j}^{\min }$ denotes the offered QoS in service class $j$ at zero load level, i.e., $c_{j}^{\min }=c_{j}(0)$ then

$$
\forall i, j \in[1, n], \quad i<j: \quad c_{i}^{\min }>c_{j}^{\min } .
$$

Assuming that the service class with better QoS is more expensive, each user wants to choose the cheapest but satisfactory service class, i.e., the service class where the offered QoS meets the user's requirement. Furthermore, let $Q_{i}$ denote the index of the preferred service class, which is the cheapest satisfactory service class of user $i$ :

$$
Q_{i}=\min _{j \in[1, n]}\left\{j: c_{j}^{\min } \leq \Theta_{i}\right\},
$$

where $\Theta_{i}$ denotes the QoS requirement of the user.

The basic model of Park et al. assumes that all of the users can afford the classes above $Q_{i}$, since the price is not an issue there [5]. It is more realistic if we assume that each user sets an affordable maximum price. Introducing this limit obviously requires a sophisticated user database and algorithms to estimate the affordable prices of representative user groups. However, using price limit results in a more general framework for the capacity allocation model.

Let $\Pi_{i}$ denote the maximum price of the bandwidth unit which can be paid by user $i$. Let $p_{j}$ denote the price of the bandwidth unit in service class $j$. Assuming that (7) holds, and better QoS is more expensive as

$$
\forall i, j \in[1, n], \quad i<j: \quad p_{i}<p_{j},
$$

we can define $P_{i}$ as the index of the most expensive, but affordable satisfactory service class for user $i$ :

$$
P_{i}=\max _{j \in[1, n]}\left\{j: \quad j \geq Q_{i}, \quad p_{j} \leq \Pi_{i}\right\}
$$

Those users, whose $P_{i}$ does not exist (i.e., they cannot afford the cheapest but satisfactory service class) are not considered in the calculations. The modified utility function of the users is thus

$$
U_{i j}^{\mathrm{p}}=\left\{\begin{array}{ll}
\lambda_{i j}, & \text { if } \Theta_{i} \geq c_{j}\left(q_{j}\right) \wedge \Pi_{i} \geq p_{j} \\
0, & \text { otherwise }
\end{array} .\right.
$$

Hence, we can formulate primary bandwidth requirement values for all of the possible service class intervals as

$$
D_{[i, j]}=\sum_{k: Q_{k}=i \wedge P_{k}=j} \lambda_{k},
$$

which means the total bandwidth requirement of users whose preferred service class is the $i^{\text {th }}$ one but can afford the service classes up to the $j^{\text {th }}$ one.

Using $D_{[i, j]}$ values, the network dimensioning problem can be handled without looking at the individual expectations of the users.

\section{B. Domain control scenarios}

We apply the model to a domain offering different service classes, e.g., a DiffServ domain, through which different areas can be reached (Fig. 1).

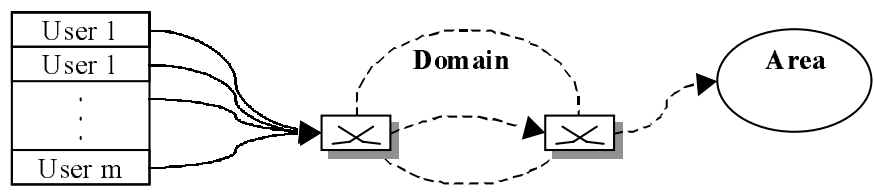

Fig. 1. Service provisioning scenario

Three basic domain control scenarios can be considered in the aspect of admission control and service mapping.

No domain control. In case of lack of any control on the network, the users are free to choose any of the offered service classes (e.g., choosing an appropriate DSCP in DiffServ networks [2]). Note that without admission control, overloading of the classes is allowed degrading the experienced quality in that service class for all the involved users.

Admission control (AC) on the edge. Introducing admission control at the edge of the domain, the overloading of the classes can be avoided. It means that a user's request is denied if there is no room to accommodate its traffic in the service 
class. This prevents the already accepted demands to get inadequate service. However, it is still the user's task to choose the appropriate service class.

Centralized domain control (CDC). Using a centralized domain control module, admission control can be introduced in a centralized manner. Moreover, the module is aware of the available network resources within the domain, and hence can portion the capacities of the service classes among the users. Once a user claims a bandwidth, quality and price requirement, the domain controller can map it to an appropriate service class, executing the service mapping task on behalf of the users. In DiffServ terminology, the Bandwidth Broker [9] can be used to perform these tasks.

Table I shows a summary of the possible domain control scenarios.

\begin{tabular}{r|l|l|l} 
& $\begin{array}{l}\text { No domain } \\
\text { control }\end{array}$ & $A C$ & $C D C$ \\
\hline Service Mapping & user & user & controller \\
\hline Overload & allowed no & no \\
TABLE I
\end{tabular}

DOMAIN CONTROL SCENARIOS

\section{DIMENSIONING STRATEGIES}

The task of the dimensioning strategies is to portion a given available capacity among the previously defined service classes.

A more realistic model is where a certain connection is assumed among the capacity, QoS and the price, e.g., providing the required bandwidth at a higher quality level requires more physical capacity. In this case, the quality improvement is solved by allocating more capacity than the actual bandwidth demand at the same tariff, which results in a certain price differentiation as well.

However, in the introduced model we assume that the operator has the ability to provide any portion of the total capacity in all of the classes, i.e., all of the capacity can be allocated to the lowest or to the highest capacity class. Obviously, offering higher QoS requires more network resources which is covered by the higher income.

Assuming that (11) holds, if there is enough capacity, demands falling into $D_{[i, j]}$ can be satisfied between any service class from class $i$ to class $j$ without degrading the utility experienced by the users. However, without CDC, as the service mapping is done by the users, they choose the cheapest but satisfactory service class as preferred. Hence, while formulating a dimensioning strategy, the service mapping becomes an important issue.

The first strategy being introduced follows the expected intentions of the users and remains effective in case of the lack of $\mathrm{CDC}$, while the second one strongly requires it. In the following sections, the methods are described in details. Their summary is drawn on Fig. 2.
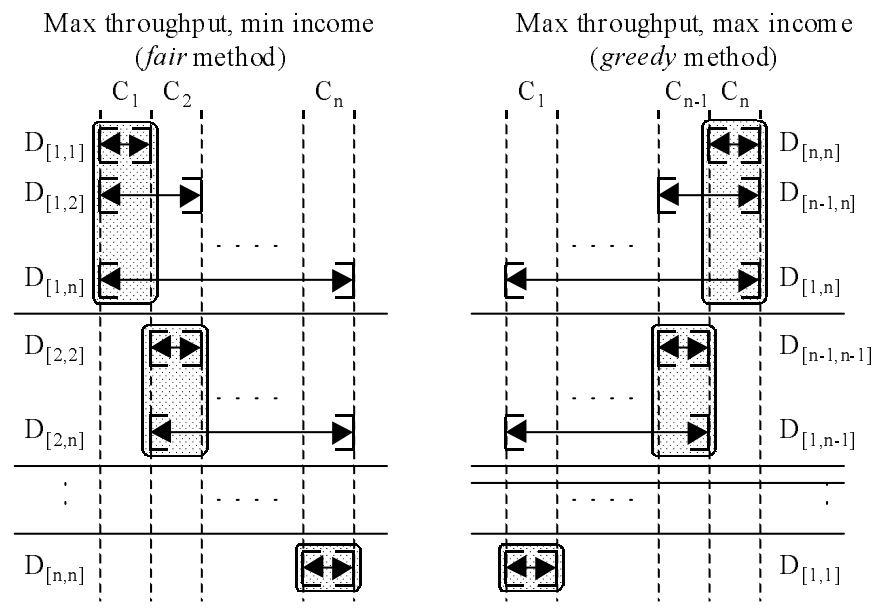

Fig. 2. Dimensioning strategies

\section{A. Throughput maximization without CDC}

Without CDC, the users are allowed to choose among the offered service classes. Therefore, while dimensioning the classes it has to be taken into account that the users try to choose the preferred service class first $\left(Q_{i}\right)$. Hence, if the sum of the available capacity is given by $C$, the first service class can be set to

$$
C_{1}=\min \left(C, \sum_{k=1}^{n} D_{[1, k]}\right)
$$

That is, summing up the demands which can be satisfied in the first class, $C_{1}$ can serve all of the demands which choose it as preferred, if there is ample capacity to share. In general, the dimensioning equation of class $j$ is given by

$$
C_{j}=\min \left(C-\sum_{k=1}^{j-1} C_{k}, \sum_{k=j}^{n} D_{[j, k]}\right), \quad j=2, \ldots, n .
$$

Note, that the remaining capacity which can be used for dimensioning class $j$ is less than $C$ by the sum of the previously allocated capacities.

This dimensioning method leads to situations where the throughput is maximized (i.e., all the available capacities are utilized), but the overall income of the network operator is the lowest. Therefore, the method can be considered as fair for the users.

\section{B. Income maximization}

If the goal is to maximize the total income, the classes can be dimensioned by stressing the users to choose their most expensive but affordable class, and try to serve as many of them as possible (hence the throughput is maximized as well). This results in a greedy method.

In this case, the method starts with the most expensive service class. Similarly to the previous method, the capacity of 
class $n$ can be calculated by summing up the demands which can be satisfied in that class:

$$
C_{n}=\min \left(C, \sum_{k=1}^{n} D_{[k, n]}\right) \text {. }
$$

Generalizing the method to the other classes, we get

$$
C_{j}=\min \left(C-\sum_{k=j+1}^{n} C_{k}, \sum_{k=1}^{j} D_{[k, j]}\right), \quad j=1, \ldots, n-1 .
$$

Note, that using this method, CDC is essential. Without centralized service mapping, users would not choose the most expensive class. However, centralized service mapping leads to Nash equilibrium as well. Recall, that the users step (i.e., choose another service class) only if they can improve their individual lots. After sizing the capacities as introduced and forcing the users to choose the most expensive class, they could neither choose another class by improving the experienced utility, nor choose a cheaper class. The reason for this is that the capacity dimensioning method ensures that no free capacity remains in the cheaper classes.

\section{Evaluation}

In the cases when the resource allocation mechanism takes place without any control in the domain (i.e., no admission control is performed and the service mapping is done by the users), the overall gained utility cannot be estimated precisely. Allowing the overloading of the network results in situations when not only the demands which cannot fit into the chosen class but also the whole class is suffering. Considering this phenomenon, bounds can be given for the total utility experienced by the users in different capacity configurations [5].

For the domains, where at least AC is performed, the introduced capacity allocation methods can be used. For evaluating them, we used computer simulations and have formulated five service classes offering different QoS, and 300 users with diverse demands in terms of bandwidth, QoS and price. The bandwidth demands ranged between 0.5 and $25 \mathrm{Mbit} / \mathrm{s}$, and the maximum price values were set between 10 and 60 monetary units. The prices of the bandwidth units $(1 \mathrm{Mbit} / \mathrm{s})$ in the offered service classes were set to 10, 20, 30, 40, 50 monetary units, respectively. The total capacity is set from 0 up to 700 Mbit/s. Fig. 3 shows the expected income depending on the total capacity to share, using different dimensioning methods.

Using the fair allocation method, which considers the intentions of the users (they prefer choosing the cheapest but satisfactory classes), curve "Fair" shows the expected income. That is, this result can be reached without introducing CDC.

Similarly, the curve "Greedy + CDC" shows a theoretic maximum of the expected income since using the greedy method with domain control the users are forced to choose the maximum affordable service class, and the throughput is also maximized.

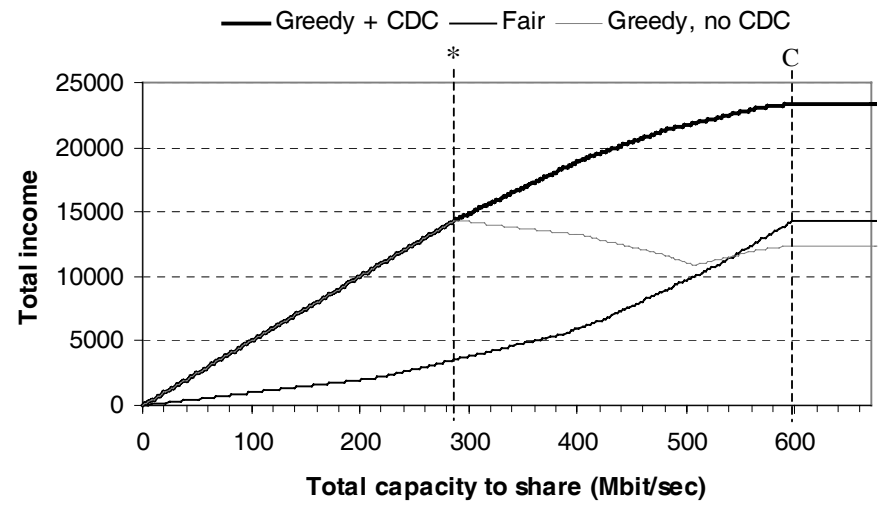

Fig. 3. Dimensioning methods with admission control

However, loosing the ability to force the users to choose the most expensive classes (i.e., the service mapping is performed on the user side, only AC is applied), the greedy algorithm can even perform worse than the fair one. That is, at a certain point, the greedy dimensioning method can result in a lower income, as it is shown on curve "Greedy, no CDC". Moreover, this curve shows a decrease in the income while increasing the available capacity which is quite interesting. The reason for this phenomenon is that in this example the service mapping is performed on the user's side in the most "unfair" way: while utilizing service class $j$, the richest users step first, who may afford expensive service classes as well (their $P_{i}$ value is the highest). Since the goal of the greedy method is to reach the highest income, it allocates capacity to the most expensive class first. $C_{n}$ is set based on demands $D_{[i, n]}$ (see (15)) and the capacities of the lower classes are set to zero. Once $C_{n}$ satisfies all the demands falling into $D_{[i, n]}($ see (*) in Fig. 3), the available free capacity is allocated to $C_{n-1}$, based on $D_{[i, n-1]}$ (see (16)). Obviously, the richest users choose the cheapest but satisfactory class, which is the $(n-1)^{\text {th }}$ one in this case. Therefore, their demands are satisfied on a lower price, extruding the users whose $P_{i}$ value is $(n-1)$ at the same time which results in an underutilization of service class $n$. Hence, because of the unfair service mapping, increasing the overall capacity results in a decrease in income and causes underutilization of the available resources.

Without any control on the network, the resulted income can be worse applying the fair method, too. Without AC, it is possible to overload the service classes which results in downgrading the quality of the whole class. In Fig. 4, simulation results are given for both of the dimensioning methods without any control. Note, that the big jumps in the curves are the results of the overloading. That is, when all the demands falling into a service class are satisfied, they get the same good quality at the same time. For easing the comparison, the thin curves shows the theoretically achievable maximum values using the greedy and the fair methods as it is shown in Fig. 3. 


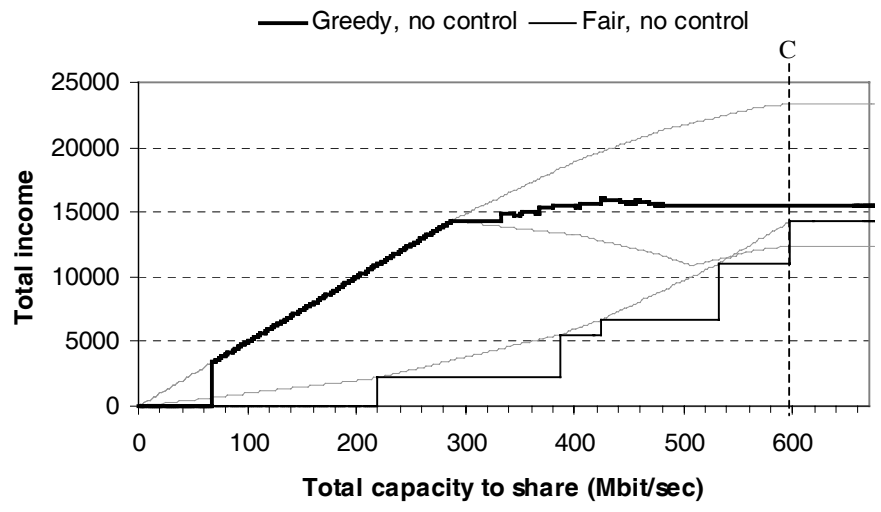

Fig. 4. Dimensioning methods without any domain control

\section{CONCLUSIONS}

Besides technical aspects, offering QoS in an environment where service differentiation is introduced requires the analysis of the achievable situations in terms of total throughput, total experienced utility or income. We investigated configurations where users claim QoS, bandwidth and price requirements against the network, which offers different service classes on different price levels. We used a game theoretic approach for the analysis, considering the users as selfish and non-cooperative players competing for network resources.

By analyzing service class dimensioning strategies in different domain control scenarios we have shown a fair dimensioning method which takes account of the user's intentions while maximizing the expected throughput. Also, a greedy method is given which maximizes the expected income of the operator (and thus the throughput as well). However, it requires strict domain control to lead the system into Nash equilibrium where the users get the quality at the highest price, since within a certain configuration more equilibrium situations can exist.

Simulations were also given on the dimensioning methods, showing that loosing the strict domain control (i.e., only AC is performed), the greedy method can perform worse than the fair one. Moreover, it shows an unacceptable behavior since increasing the available capacity resulted in a decrease in the expected income and caused underutilization of the network. Besides this, examples show the way the methods work in a system where no control is performed on the network domain at all.

Investigating other network dimensioning strategies, where a strict correlation exists among the capacity, QoS and price are the topic of further studies.

\section{REFERENCES}

[1] T. Basar and G. J. Olsder, Dynamic Noncooperative Game Theory, Academic Press, second edition, 1995.

[2] S. Blake et al., An Architecture for Differentiated Service, IETF RFC, rfc2475.txt 1998.

[3] B. Braden, Ed., et. al., Resource Reservation Protocol (RSVP) - Version 1 Functional Specification IETF RFC, rfc2205.txt, 1997.

[4] J.W. Friedman, Game Theory with Applications to Economics, Oxford University Press, 1986.

[5] P. Füzesi, A. Vidács, Investigating Nash Equilibria in Differentiated Services Networks, submitted to Infocom 2002, New York.

[6] R. J. Gibbens, Control and Pricing for Communication Networks, Research Report, Statistical Laboratory, University of Cambridge, 1999.

[7] Y. Korilis, A. Lazar and A. Orda, Architecting Noncooperative Networks, IEEE J. Select. Areas Commun., 13(7):1241-1251, 1995.

[8] Y. Korilis, A. Lazar and A. Orda, Achieving Network Optima Using Stackelberg Routing Strategies, IEEE/ACM Trans. Networking, 5(1):161-173, 1997.

[9] K. Nichols, V. Jacobson, L. Zhang, A Two-bit Differentiated Services Architecture for the Internet, IETF RFC, rfc2638.txt

[10] K. Park, M. Sitharam and S. Chen, Quality of Service Provision in Noncooperative Networks: Heterogenous Preferences, Multi-Dimensional QoS Vectors, and Burstiness, Technical Report CSD-TR-97-039A, Dept. of Computer Sciences, Purdue University, 1998.

[11] H. Ren, K. Park, Toward a Theory of Differentiated Services, In Proc. IEEE/IFIP International Workshop on Quality of Service, pp. 211-220, 2000

[12] J.W. Roberts, Engineering for Quality of Service, Draft chapter for a book "Self-similar Network Traffic and Performance Evaluation", edited by K. Park and W. Willinger, 1998.

[13] J. Wroclawski, The Use of RSVP with IETF Integrated Services, IETF RFC, rfc2210.txt 